\title{
Estrategias de gestión ambiental para el manejo y disposición final del catalizador gastado de la unidad de craqueo catalítico fluidizado (FCC) generado en una refinería estatal de Ecuador
}

\section{Strategies of environmental management for the management and final disposal of the spent catalyst of the fluidized catalytic cracking unit (FCC) generated in a state refinery in Ecuador}

Suárez Daysi

Coral Katty

Gallegos Walberto

Universidad Internacional SEK, Ecuador

Tapia Diego

Investigador independiente, Ecuador

Autor por correspondencia: walberto.gallegos@uisek.edu.ec

Fecha de recepción: 6 de Febrero de 2017 - Fecha de aceptación: 28 de Abril de 2017

Resumen: La Refinería Estatal Esmeraldas "REE" cuenta con una unidad de Craqueo Catalítico Fluidizado (FCC), cuyo objetivo es la producción de combustible de alto octano a partir del Gasóleo y crudo reducido de la unidad de Destilación al Vacío (VGO). Durante la operación, el catalizador es presente en las reacciones del proceso. Consecuentemente, el catalizador disminuye su área superficial, aumenta la densidad aparente, y se convierte en lo que se conoce como catalizador gastado, que no puede ser regenerado y es desechado como residuo. Se caracterizó al catalizador gastado de la FCC mediante los parámetros físico químicos: ABD, DSP, metales en peso seco y características CRTIB. La característica de toxicidad ambiental se definió mediante ensayo de lixiviación (EPA 1311). Se aplicó la metodología IBR según API 581 y la evaluación de impactos ambientales según Leopold. Se determinó que el catalizador gastado de craqueo catalítico evaluado, no excede en ninguno de los límites máximos permisibles de las características CRTIB, para considerarse como un residuo peligroso. Según la prueba estadística no paramétrica de Krustal Wallis ( $\mathrm{p}<0,0001$ ), se plantea la reposición (make up) del catalizador como medida de prevención en la fuente, para mantener un bajo contenido de metales durante la alimentación y la re- utilización del catalizador gastado como materia prima en cementeras para su aprovechamiento y/o valorización.

Palabras clave: FCC; catalizador; metales; VGO; CRTIB

Abstract: The Esmeraldas State Refinery "REE" has a Fluidized Catalytic Cracking (FCC) unit, whose objective is the production of high octane fuel from Diesel and reduced crude from the Vacuum Distillation (VGO) unit. During operation, the catalyst is deactivated due to heavy metal poisoning and coke fouling present in the process reactions. Consequently, the catalyst decreases its surface area, increases bulk density, and becomes what is known as spent catalyst, which cannot be regenerated and is discarded as waste. The spent catalyst of the FCC was characterized by the physical chemical parameters: ABD, DSP, dry weight metals and CRTIB characteristics. The 
environmental toxicity characteristic was defined by leach test (EPA 1311). We applied the IBR methodology according to API 581 and the environmental impact assessment according to Leopold. It was determined that the spent catalytic cracking catalyst evaluated does not exceed at any of the maximum allowable limits of the CRTIB characteristics to be considered as a hazardous waste. According to the Krustal Wallis non-parametric statistical test ( $p<0.0001$ ), the make-up of the catalyst is proposed as a prevention measure at source to maintain a low metal content during feed and re-use of the catalyst spent as raw material in cement for its use and / or valorization.

Key words: FCC; catalyst metals; VGO; CRTIB

\section{Introducción}

La generación de residuos asociados con catalizadores gastados empleados en diferentes operaciones unitarias en la industria de la refinación del petróleo, constituye un importante problema ambiental debido a la especialización en los metales que componen los catalizadores, circunstancia que complica la valoración (Castells, 2012).

Durante la operación de la unidad de craqueo catalítico fluidizado, el "catalizador zeolítico" sufre envenenamiento por depósitos de metales alcalinos y pesados contenidos en el procesamiento de crudo. Como consecuencia, el catalizador disminuye su área superficial, aumenta la densidad aparente y se convierte en lo que se conoce como catalizador gastado, que no puede ser regenerado; por lo tanto es reemplazado por un catalizador fresco para continuar el proceso (Sedrán, 2006). Los catalizadores de FCC son uso común para la conversión de gasóleo (VGO) en productos de mayor octanaje. En el mundo, se estima una generación de 840.000 toneladas de catalizador agotado (Letzsch, 2014) como residuo.

Históricamente, los catalizadores gastados se envían a sitios de disposición final de residuos sólidos. Aunque cada vez son más abundantes las tecnologías para el aprovechamiento y/o valorización de catalizadores, con el fin de evitar el depósito incontrolado en vertederos (Ferella, 2016). En Ecuador, los catalizadores agotados provenientes del proceso de refinación de petróleo y coque están catalogados como desechos peligrosos por su característica CRITIB de toxicidad ambiental conforme el Listado Listado Nacional de Desechos Peligrosos por fuente específica del Acuerdo Ministerial No 142 del Ministerio del Ambiente de Ecuador.

No obstante, los principales métodos de valorización de catalizadores agotados de FCC reportados (Tabla 1), implican su uso como materia prima para cementeras y su incorporación como adición al cemento Portland, junto con otros materiales para la fabricación de fritas cerámicas y como aditivo inorgánico en la composición de las suspensiones que se aplican para la obtención de los recubrimientos vidriados de las piezas de pavimento gresificado (Al-Dhamri \& Melghit, 2010). Así mismo, podría utilizarse, en lugar de caolín, para la obtención de zeolitas.

Tabla 1. Estrategias de valorización y reutilización de catalizadores agotados

\begin{tabular}{lccc}
\hline Tipo de catalizador agotado & \multicolumn{3}{c}{ Estrategia de gestión ambiental } \\
\cline { 2 - 4 } & $\begin{array}{c}\text { Recuperación de } \\
\text { metales }\end{array}$ & $\begin{array}{c}\text { Materia Prima en } \\
\text { cementeras }\end{array}$ & $\begin{array}{c}\text { Nutrientes de } \\
\text { fósforo }\end{array}$ \\
\hline Polimerización & No & No & Sí \\
\hline
\end{tabular}




\begin{tabular}{lccc}
\hline Craqueo catalítico (FCC) & Sí & Sí & No \\
\hline Reformado catalítico & Sí & No & No \\
\hline Hidrocracking & Sí & No & No \\
\hline Hidrotratamiento & Sí & Sí & No \\
\hline Óxido de plomo & Sí & Sí & No \\
\hline Alúmina activa en alquilación & No & No & No \\
\hline $\begin{array}{l}\text { Alúmina activa del proceso } \\
\text { Clauss }\end{array}$ & Sí & No & No \\
\hline Cloruro de cobre & Sí & Sí & No \\
\hline Conversión en cloruros & & No & No
\end{tabular}

Fuente: (Castells, 2012)

El presente trabajo se enfoca al planteamiento de estrategias ambientalmente sustentables de gestión ambiental para un adecuado manejo y disposición final del catalizador gastado de la FCC generado en la Refinería Estatal de Esmeraldas.

\section{Materiales y métodos}

\section{Muestreo del catalizador gastado}

Se procedió a realizar un muestreo no probabilístico del Catalizador gastado proveniente de la tolva F-V2 de la Unidad de Craqueo Catalítico FCC en Refinería Esmeraldas. La muestra fue puntual de $1 \mathrm{~kg} / \mathrm{mes}$. Se empleó el método del cuarteo según la norma (SEMANART NMX 015-1985) para obtener una muestra homogénea del catalizador gastado.

\section{Caracterización del catalizador gastado}

La densidad volumétrica aparente (ABD) se determinó según la norma NTE INEN 0856; 2010. Se determinó la distribución del tamaño de partícula (DSP) según la metodología estandarizada de tamices Tyler. El análisis de metales pesados en peso seco fue realizado según la norma EPA 6020 A y el contenido de Hidrocarburos Totales de petróleo, según el método EPA 8015 D

\section{Determinación de características de peligrosidad CRTIB}

Se efectuaron pruebas fisicoquímicas para determinar las características de peligrosidad CRTIB del catalizador, según los métodos normalizados de la norma NOM-052-Semarnat/93.

\section{Definición de toxicidad ambiental}


Para definir la toxicidad del catalizador agotado se adoptó la metodología establecida por la norma EPA 1311, de acuerdo a la concentración de los metales pesados considerados como tóxicos en concentraciones reducidas: $\mathrm{Ag}, \mathrm{Ba}, \mathrm{As}, \mathrm{Cd}, \mathrm{Cr}, \mathrm{Hg}, \mathrm{Pb}, \mathrm{Se}(\mathrm{EPA}, 2003)$ y posteriormente se compararon con los límites máximos permisibles de la norma técnica internacional NOM-052-SEMARNAT/93 aceptada por el Ministerio de Ambiente de Ecuador.

El ensayo de toxicidad TCLP fue realizado en un Laboratorio acreditado por la Sociedad de Acreditación Ecuatoriana (SAE).

\section{Inspección Basada en Riesgos (IBR)}

Se empleó la metodología de Inspección Basada en Riesgo (IBR) según la norma API 581 mediante el Software RiskWASE 5.

Tabla 2. Criterios para determinar el nivel de Riesgo en la matriz IBR

\begin{tabular}{ccccc}
\hline & Factor Probabilidad & & Consecuencias $\left(\right.$ pies $\left.^{2}\right)$ & Riesgo \\
\hline 1 & $\mathrm{FP} \leq 1$ & $\mathrm{~A}$ & $\mathrm{C} \leq 10$ & BAJO \\
\hline 2 & $1<\mathrm{FP} \leq 10$ & $\mathrm{~B}$ & $10<\mathrm{C} \leq 100$ & BAJO \\
\hline 3 & $10<\mathrm{FP} \leq 100$ & $\mathrm{C}$ & $100<\mathrm{C} \leq 1000$ & MEDIO \\
\hline 4 & $100<\mathrm{FP} \leq 1000$ & $\mathrm{D}$ & $1000<\mathrm{C} \leq 10000$ & MEDIO ALTO \\
\hline 5 & $\mathrm{FP}>10$ & $\mathrm{E}$ & $\mathrm{C}>100$ & ALTO \\
\hline
\end{tabular}

Fuente: (API 581, 2000)

\section{Evaluación de impactos ambientales}

Se evaluaron los impactos ambientales asociados a las estrategias de manejo y disposición final según los criterios de la Matriz Modificada de Leopold o matriz de Importancia que permitió identificar las acciones y/o actividades de los procesos a través de la importancia del impacto generado.

\section{Evaluación de riesgos de exposición del manejo del catalizador gastado}

Para estimar los riesgos de exposición propias del uso del catalizador gastado, tal como la inhalación de material particulado, se comparó la concentración de los contaminantes con valores de exposición (SSLs, por sus siglas del inglés Soil Screening Levels) de la (USEPA, 1996) Se utilizó como ruta de exposición la inhalación del material particulado por considerarse la más significativa en función del manejo del catalizador. En casos en los cuales no se reportan límites para inhalación, se utilizaron los correspondientes a ingestión.

\section{Resultados y discusión}

\section{Caracterización del catalizador gastado de la FCC}

La densidad volumétrica del catalizador gastado fue de $\pm 0,81 \mathrm{~g} / \mathrm{mL}$ en comparación con el catalizador fresco, cuya densidad volumétrica fue de \pm 0,80 g/mL. Según (Sadeghbeigi, 2000) 
la densidad aparente está relacionada con la fluidización y circulación del catalizador, de esta manera si el contenido de zeolita es alto, el catalizador es menos denso.

En relación con la granulometría del catalizador gastado de FCC, se determinó que el $76,23 \%$ del contenido del catalizador gastado fue retenido en el cedazo de 75 um ( $\mathrm{N}^{\circ}$ malla 200), mientras que el 17,86\% fue retenido en el tamiz de 45 um $\left(\mathrm{N}^{\circ}\right.$ malla 325).

\section{Metales en peso seco}

Se determinó que los principales contaminantes metálicos del catalizador gastado son Vanadio (382 mg/kg) y Níquel $(75 \mathrm{mg} / \mathrm{kg}$ ). Conforme lo expuesto por (Mitchell, Hoffman, \& Moore, 2003) el Vanadio tiene una fuerte tendencia destructiva hacia la zeolita, mientras que el Níquel provoca reacciones de des hidrogenación no deseadas que producen hidrógeno y coque.

\section{Hidrocarburos totales de petróleo.}

El contenido de hidrocarburos totales del catalizador gastado de FCC fue inferior al límite de detección del método analítico. Aun cuando los hidrocarburos totales no se utilizan para evaluar la toxicidad ambiental, su concentración permitió estimar la presencia o no de TPH’s en el catalizador gastado de FCC. De esta manera, se pudo evidenciar que el catalizador gastado de la FCC no está contaminado con Hidrocarburos Totales de Petróleo.

\section{Determinación de características de peligrosidad CRTIB}

Corrosividad: la medida del $\mathrm{pH}$ del catalizador gastado fue de $\pm 5,3$, encontrándose dentro de los límites permisibles ( 2 a 12,5 unidades de $\mathrm{pH}$ ) para considerar a un residuo sólido como peligroso según la norma (NOM-052-SEMARNAT/93); descartándose así la característica de corrosividad (C) para el catalizador gastado de FCC.

Velocidad de corrosión: conforme los resultados obtenidos, se emplearon los cupones de corrosión de latón- estaño y acero. La velocidad de corrosión para el coupon 1 fue de 3,70 milímetros por año (mmy) y para el coupon 2 de 3,720 milímetros por año (mmy). Según la norma (NOM-052-SEMARNAT/93), se considera un residuo como corrosivo cuando es capaz de corroer el acero, a una velocidad de corrosión de 6,35 milímetros o más por año. En ambos casos no se superaron los límites máximos permisibles para considerar a un residuo como peligroso.

Reactividad: se evidenció que el catalizador gastado no reacciona violentamente formando gases, vapores o humos, por lo que no es capaz de provocar una detonación violenta, bajo condiciones normales $\left(25^{\circ} \mathrm{C}\right.$ y $\left.1 \mathrm{~atm}\right)$.

\section{Definición de toxicidad ambiental}

La concentración de los metales en lixiviado (As, Ba, Cd, Hg, Ni, Ag, Pb, Se, Cr, V) estuvo por debajo de los límites permisibles de la norma EPA 1311 y NOM-052-

SEMARNAT/93 (Tabla 3). 
Cabe destacar que no se encontraron límites para el Vanadio en lixiviado en las normas (NOM-052-SEMARNAT/93) y EPA 1311 (EEUU). Por ello se comparó con los límites permisibles de lixiviados para la disposición final de lodos con impermeabilización en la base del Reglamento RAOHE-1215 para operaciones hidrocarburíferas en Ecuador. A manera de referencia, la concentración de Vanadio en el lixiviado no debe superar 2 mg/L (RAOHE, 2001).

El resultado del análisis de concentración de Vanadio en el lixiviado del catalizador gastado de FCC, es menor, en ambos casos, (Gráfico 1).

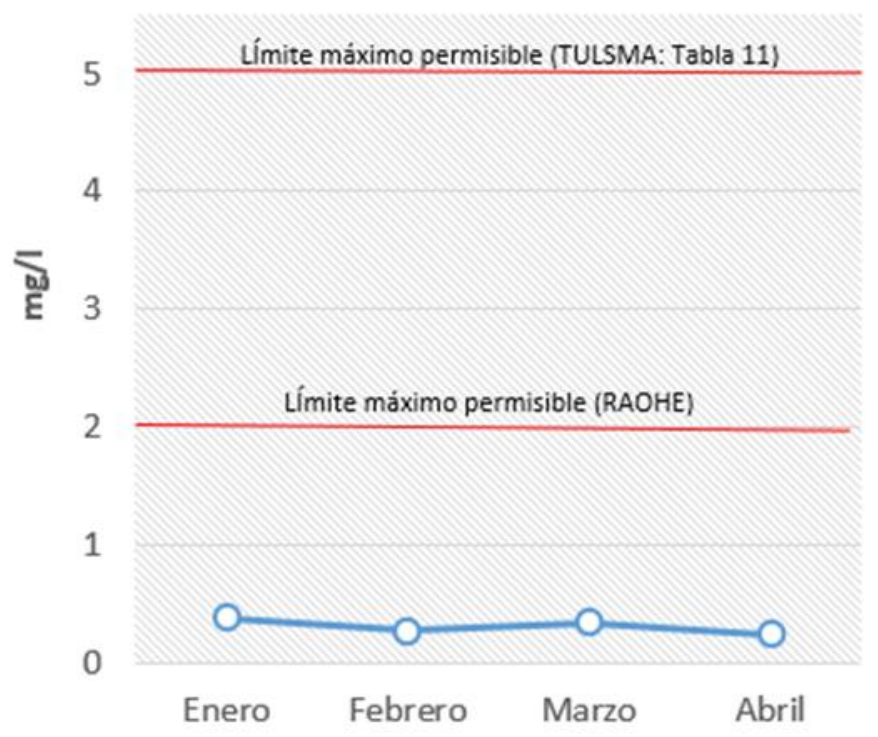

Gráfico 1. Concentración de Vanadio (mg/L) en el lixiviado del catalizador gastado de FCC periodo de enero a abril 2016.

En relación a la concentración del Níquel en el lixiviado, su concentración fue inferior al límite máximo permisible según la norma EPA 1311 para metales en lixiviado. La norma EPA 1311 establece el máximo límite permisible de $5 \mathrm{mg} / \mathrm{L}$ para Níquel. No obstante, según los límites de descarga al sistema de alcantarillado público establecidos en el Texto Unificado de Legislación Ambiental (TULSMA) en relación al Níquel, la concentración no debe ser superior a $2 \mathrm{mg} / \mathrm{L}$, siendo este valor del TULSMA superior a la concentración de Níquel en el lixiviado del catalizador gastado de FCC (Gráfico 2). 


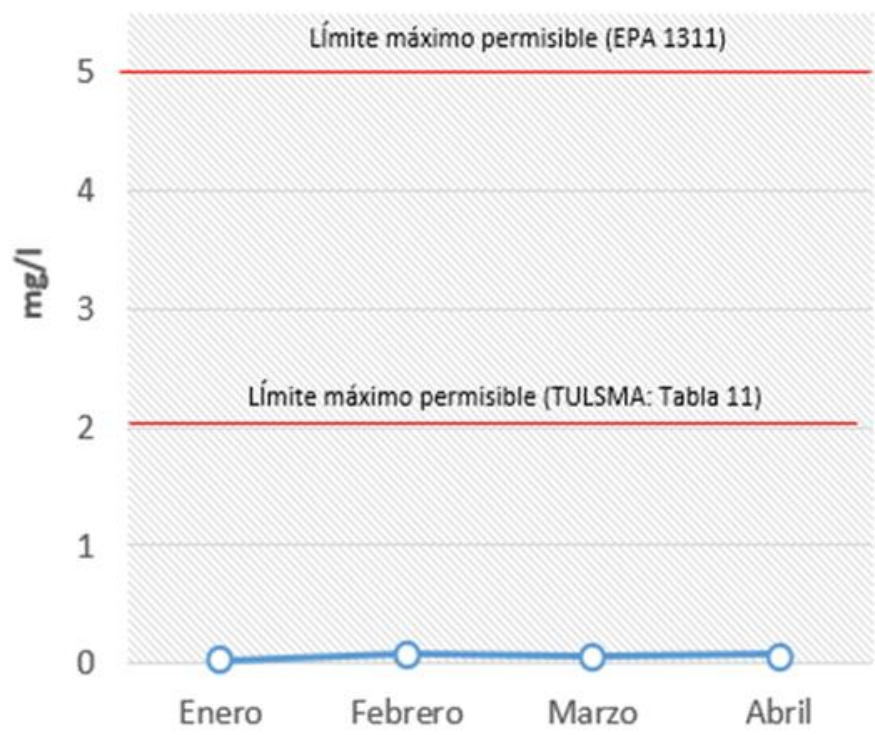

Gráfico 2. Concentración de Níquel (mg/L) en el lixiviado del catalizador gastado de FCC periodo enero a abril 2016.

Por lo expuesto, los resultados obtenidos demuestran que el catalizador gastado de FCC evaluado, no excede ninguno de los límites máximos en lixiviados, para considerar un residuo como tóxico - peligroso (T). Sin embargo, debido al volumen de generación de 2 ton/día, equivalente a 60 ton/mes, el catalizador gastado de la FCC podría considerarse como un Desecho

Especial, cuyos límites de concentración establecidos no superan la normativa ambiental.

Tabla 3. Resultados de monitoreo de metales en lixiviado

\begin{tabular}{|c|c|c|c|c|c|c|c|}
\hline Metales & Unidad & LMP & $\begin{array}{l}\text { CAT } \\
\text { GAS-01 } \\
18 / 1 / 2016\end{array}$ & $\begin{array}{l}\text { CAT } \\
\text { GAS-02 } \\
22 / 2 / 2016\end{array}$ & $\begin{array}{l}\text { CAT } \\
\text { GAS-03 } \\
18 / 3 / 2016\end{array}$ & $\begin{array}{l}\text { CAT GAS- } \\
04 \\
22 / 4 / 2016\end{array}$ & $\begin{array}{l}\text { Cumple } \\
\text { Normativa } \\
\text { ambiental }\end{array}$ \\
\hline Arsénico & $\mathrm{mg} / \mathrm{L}$ & $5 *$ & $<0,005$ & $<0,005$ & $<0,005$ & $<0,005$ & SI \\
\hline Bario & $\mathrm{mg} / \mathrm{L}$ & $100 *$ & 0,17 & 0,14 & 0,15 & 0,16 & SI \\
\hline Cadmio & $\mathrm{mg} / \mathrm{L}$ & $1 *$ & $<0,001$ & $<0,001$ & $<0,001$ & $<0,001$ & SI \\
\hline Mercurio & $\mathrm{mg} / \mathrm{L}$ & $0,2 *$ & $<0,001$ & $<0,001$ & $<0,001$ & $<0,001$ & SI \\
\hline Níquel & $\mathrm{mg} / \mathrm{L}$ & $5 *$ & 0,032 & 0,09 & 0,057 & 0,08 & SI \\
\hline Plata & $\mathrm{mg} / \mathrm{L}$ & $5 *$ & $<0,001$ & $<0,001$ & $<0,001$ & $<0,001$ & SI \\
\hline Plomo & $\mathrm{mg} / \mathrm{L}$ & $5 *$ & $<0,005$ & $<0,005$ & $<0,005$ & 0,0064 & SI \\
\hline Selenio & $\mathrm{mg} / \mathrm{L}$ & $1 *$ & 0,014 & 0,042 & 0,022 & 0,024 & SI \\
\hline Vanadio & $\mathrm{mg} / \mathrm{L}$ & $<2 * *$ & 0,38 & 0,27 & 0,33 & 0,24 & SI \\
\hline $\begin{array}{c}\text { Cromo } \\
\text { VI }\end{array}$ & $\mathrm{mg} / \mathrm{L}$ & $5^{*}$ & $<0,02$ & $<0,02$ & $<0,02$ & $<0,02$ & SI \\
\hline
\end{tabular}

Fuente: Reportes GRUENTEC 1601204-S001, 1602233-DS001, 1603369-DS001, 1605008DS001.

* LMP EPA 1311: Límites máximos permisibles para los constituyentes tóxicos en el extracto PECT (NOM-052SEMARNAT/93).

** LMP: Límites permisibles de lixiviados para la disposición final de lodos (RAOHE-1215) con impermeabilización en la base. 


\section{Inspección basada en riesgos (IBR) según la norma API 581.}

Para definir la probabilidad de riesgo en el manejo del catalizador gastado desde la tolva F-V2 se empleó la metodología IBR de acuerdo a la norma API 581. Según los resultados obtenidos mediante el software RiskWISE 5, el nivel de riesgo de fuga del contenido de catalizador gastado es bajo con una mínima probabilidad de ocurrencia. La matriz de riesgos IBR se muestra en el Gráfico 3.

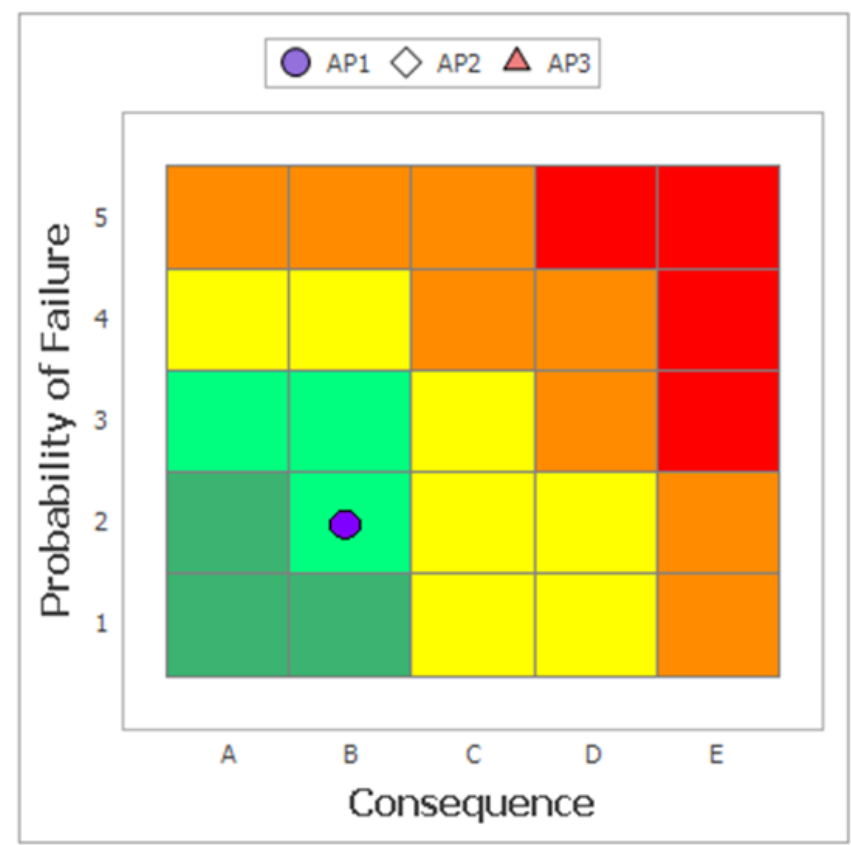

Gráfico 3. Resultado de la matriz de Riesgo (IBR)

Obtenido de: Risk Analysis-RiskWASE 5

Como se observa en el gráfico 3, la probabilidad de pérdida de material de la tolva F-V2 es baja y sin pérdidas significativas (2B). El riesgo del equipo está influenciado principalmente por las consecuencias que por las frecuencias de falla.

Según la norma API 581 (Tabla 4), todos los equipos analizados son considerados para los módulos técnicos de adelgazamiento (Thinning) y agrietamiento por corrosión bajo tensión (SCC), cuyos parámetros en el caso de la tolva F-V2 son bajos.

Tabla 4. Parámetros evaluados en la metodología IBR

\begin{tabular}{cc}
\hline Tipo de fluido & VGO (C17-C25) \\
\hline Diámetro apertura (mm/pulg) & $101,6 / 4 \mathrm{pulg}$ \\
\hline Tasa liberación (lb/seg) & 417,05 \\
\hline Duraciones de fuga & 20 minutos/4 plg \\
\hline Frecuencia de fuga (anual) & $3 \times 10^{-6}$ \\
\hline Agrietamiento por corrosión bajo tensión & Bajo: 1 \\
\hline Probabilidad (falla/año) & $3,432 \times 10^{-4}$ \\
\hline
\end{tabular}

Obtenido de: Risk Analysis-RISKWASE 5 
Según la Tabla 5, la inspección es usualmente efectiva para el 70\% de los casos. Sin embargo se requiere inspecciones bianuales para evitar pérdida de falla en el equipo estático.

Tabla 5. Resultado de la categoría de Inspección por la metodología IBR.

\begin{tabular}{ccc}
\hline Equipo & $\begin{array}{c}\text { Cantidad/ } \\
\text { Efectividad }\end{array}$ & Significado \\
\hline $\begin{array}{c}\text { Tolva FV- } \\
2\end{array}$ & $2 / \mathrm{B}$ & Usualmente efectiva. Método de inspección que identifica correctamente daños en \\
servicios en $70 \%$ de los casos.
\end{tabular}

Obtenido de: Risk Analysis-RiskWASE $5^{1}$

De acuerdo a la caracterización del catalizador gastado de la FCC y según la jerarquización en la gestión integral de los desechos peligrosos y/o especiales, se plantearon las siguientes estrategias: E1. Reposición (make up) del catalizador; E2.Recuperación de metales mediante proceso metalúrgico e hidrometalúrgico; E3. Reutilización como materia prima en cementeras y E4. Disposición final en vertedero.

Según la prueba estadística no paramétrica de Krustal Wallis (Tukey con valores significativos de ( $\mathrm{p}<0,0001)$, las estrategias con menor impacto ambiental son la E1 y E3. Se podría combinar la estrategia 1 (E1) y la estrategia 3 (E3) que siguen la misma distribución normal (Gráfico 4). La estrategia 1 (E1) permitirá mantener el perfil térmico, y un bajo contenido de metales durante la alimentación (Make-up del catalizador). Mientras que la estrategia 3 (E3), una vez que el catalizador gastado sea desechado de la tolva F-V2, podrá ser reutilizado como materia prima en cementeras.

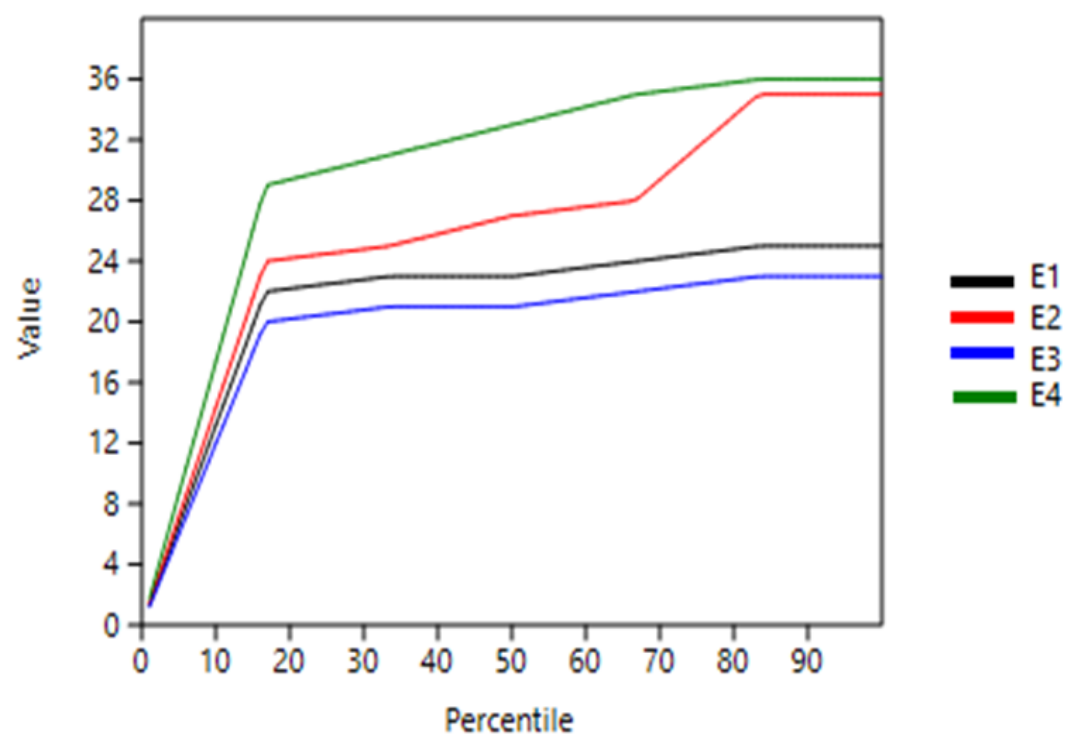

Gráfico 4. Percentiles de estrategias de gestión ambiental. Krustal -Wallis. Obtenido de: PAST 3.12

\footnotetext{
${ }^{1}$ Risk WISE 5 for Process Plant. Version 5.1.0.28105.2016

2 PAST 3.12. Scientific Data Analysis. Current version (May 2016). Copyright Hammer \& Harper. Universtity of Oslo
} 
Para la factibilidad de la aplicación de estas estrategias ambientales se consideraron los riesgos asociados a la inhalación e ingestión del material particulado, según los niveles específicos de evaluación de riesgos de exposición de la (USEPA, 1996). Según la Tabla 6, ninguno de los contaminantes evaluados superó los límites de la USEPA para considerar riesgo ecotóxico de exposición del catalizador gastado de FCC.

Tabla 6. Riesgos de exposición del catalizador gastado de FCC

\begin{tabular}{lll}
\hline Contaminante & Catalizador gastado FCC & Riesgo de exposición HSE \\
& & \multicolumn{2}{l}{ Valores en peso seco $(\mathrm{mg} / \mathrm{kg})$} & $*$ USEPA $(\mathrm{mg} / \mathrm{kg})$ \\
\hline $\mathrm{As}$ & 1,8 & 750 \\
\hline $\mathrm{Ba}$ & 125 & $6,9 \times 10^{5}$ \\
\hline $\mathrm{Cd}$ & $<0,1$ & 1800 \\
\hline $\mathrm{Cr}$ & 23 & 270 \\
\hline $\mathrm{Ag}$ & $<0,2$ & $\mathrm{NL}$ \\
\hline $\mathrm{Pb}$ & 16 & $400 * *$ \\
\hline $\mathrm{Ni}$ & 75 & 13000 \\
\hline $\mathrm{Se}$ & 10 & $390 * *$ \\
\hline $\mathrm{V}$ & 382 & 550 \\
\hline $\mathrm{Mo}$ & 0,7 & $\mathrm{NL}$ \\
\hline $\mathrm{Hg}$ & $<0,1$ & 10 \\
\hline & $*$ LP: Guía EPA. Ruta de exposición por inhalación. \\
& $* *$ Ruta de exposición por ingestión \\
& (No se reportan por inhalación)
\end{tabular}

No obstante, cabe mencionar que el catalizador en su formulación contiene óxidos inorgánicos, sales inorgánicas, en soporte inorgánico como tierras raras (p. ej oxido de lantano: $<7 \%$,) (BASF, 2016). Por lo expuesto, se consideró la posibilidad de riesgo del lantano presente en la formulación del catalizador para su reutilización como materia prima en cementeras.

Según (BASF, 2016) el lantano se emplea en catalizadores comerciales de FCC para prevenir la pérdida de aluminio y aumentar la actividad dentro de la estructura zeolítica. Aunque las normativas actuales no establecen límites permitidos de este contaminante en agua y suelos; existe preocupación de su efecto en el ambiente. Algunos estudios de ecotoxicidad en medio acuático (Zhang, 2010) (Xu \& Cheng, 2012) han reportado efectos adversos sobre el crecimiento y reproducción de organismos de prueba a concentraciones desde $10 \mathrm{mmol} \mathrm{L-1}$, valor que puede ser tomado como una referencia al evaluar la concentración de este elemento en los lixiviados.

Según la Tabla 7, la concentración de lantano $(0,468 \mathrm{mmol} / \mathrm{L})$ no superó el valor de referencia de efectos adversos ecotóxicos $(10 \mathrm{mmol} / \mathrm{L})$ para la reutilización del catalizador gastado de FCC.

Tabla 7. Límites de efectos ecotóxicos del Lantano en el catalizador gastado de la FCC 


\begin{tabular}{llll}
\hline $\begin{array}{l}\text { Metal en } \\
\text { lixiviado }\end{array}$ & $\begin{array}{l}\text { Concentración } \\
(\mathrm{mg} / \mathrm{L})\end{array}$ & $\begin{array}{l}\text { Concentración } \\
(\mathrm{mmol} / \mathrm{L})\end{array}$ & $\begin{array}{l}\text { Valor referencia para ecotoxicidad } \\
(\mathrm{mmol} / \mathrm{L})\end{array}$ \\
\hline La & 65 & 0,468 & 10 \\
\hline
\end{tabular}

Fuente: Reporte Gruentec 1605008-DS001

Con los resultados precedentes, el uso de catalizador gastado como materia prima en cementeras no representaría un impacto ambiental significativo. Según (Al-Jabri, 2013) el catalizador gastado puede sustituir hasta el $20 \%$ del cemento en morteros y mezclas de concreto.

Sin embargo, el manejo del catalizador gastado, deberá realizarse con la finalidad de minimizar la dispersión del material particulado, es decir, al mantenerlo húmedo.

\section{Conclusiones y recomendaciones}

Inicialmente, el catalizador gastado de la FCC fue catalogado por el Ministerio del Ambiente como desecho peligroso por su característica de toxicidad ambiental. Sin embargo, se evidenció que la concentración de los metales en lixiviado (As, $\mathrm{Ba}, \mathrm{Cd}, \mathrm{Hg}, \mathrm{Ni}, \mathrm{Ag}, \mathrm{Pb}, \mathrm{Se}, \mathrm{Cr}$, V) del catalizador gastado estuvo por debajo de los límites permisibles de la norma EPA 1311 y de la NOM-052-SEMARNAT/93 acogida por el Ministerio de Ambiente de Ecuador.

Se determinó que el catalizador gastado de craqueo catalítico evaluado, no excede en ninguno de los límites máximos permisibles de las características CRTIB, para considerarse como un residuo peligroso.

Debido al volumen de generación de 2 ton/día, equivalente a 60 ton/mes, el catalizador gastado de la FCC podría considerarse como un Desecho Especial, cuyos límites de concentración CRTIB establecidos no superan la normativa ambiental.

El catalizador gastado de la FCC no presenta riesgos ecotóxicos asociados a la inhalación e ingestión del material particulado, según los niveles específicos de evaluación de riesgos de exposición de la (USEPA, 1996).

De acuerdo a la jerarquización en la gestión de residuos peligrosos y especiales, se plantea la Estrategia 1. Reposición (make up) del catalizador como medida de prevención en la fuente, para mantener un bajo contenido de metales durante la alimentación.

Para el aprovechamiento adecuado del residuo según sus características físico químicas se propone la Estrategia 3. Reutilización como materia prima en cementeras con el menor impacto ambiental ( $\leq 25$ : Impacto Irrelevante) para los componentes de agua, aire, suelo y generación de residuos, considerándose la disposición final en vertedero controlado como la última alternativa para la gestión del catalizador gastado de la FCC.

\section{Bibliografía}

Adriano, D. (1992). Biogeochemistry of Trace Metals. California: Lewis Publishers. 
Akso, K. (1983). Determination of particle size distribution using micromesh sieves with square holes. Amsterdam: Akzo Chemie Nederlad.

Al-Dhamri, H., \& Melghit, K. (2010). Use of alumina spent catalyst and RFCC wastes from petroleum refinery to substitute bauxite in the preparation of Portland Clinker. Journal of Hazardous Materials, 179:852-859.

Al-Jabri, K. (2013). Potential use of FCC spent catalyst as partial replacement of cement or sand incement mortars. Constr. Build. Mater, 39:77-81.

Alonso, F., Ramírez, S., \& Ancheyta, J. (2008). Alternativas para la recuperación de metales a partir de catalizadores gastados del hidrotratamiento de hidrocarburos pesados: un caso de estudio. Rev. Int. Contam. Ambient, 24:55-69.

Amaya, J. (2005). Employing fly ash and FCC catalyser waste in recovering Chrome (III) from liquid effluent emitted by tanneries. Ingeniería e investigación, 57:39-48.

Antiohos, S. (2006). Re-use of spent catalyst fromoil-cracking refineries as supplementary cementing material. China Particuol, 4:73-76.

Aung, K., \& Ting, Y. (2005). Bioleaching of spent fluid catalytic cracking catalystusing Aspergillus niger. J. Biotechnol, 116:159-170.

Baerns, M. (2004). Basic Principles in Applied Catalysis. Berlin: Springer- Verlag Berlin Heidelberg.

Baldasano, J. (1986). El tratamiento de los residuos industriales mediante vertedero controlado. Ing. Quím, 207:63-70.

Basaldella, E. (2006). Exhausted fluid catalyticcracking catalysts as raw materials for zeolite synthesis. Appl. Catal., B:Environ, 66:186-191.

BASF. (2016). Catalizador FCC.BASF FLEX-TEC. Certificado de análisis EP Petroecuador. USA: BASF Corporation.

Bautista, B. (1999). Introducción al estudio de la contaminación del suelo por metales pesados. México: Universidad Autónoma de Yucatán.

Bayraktar, O. (2005). Bioleaching of nickel from equilibrium fluid catalytic cracking catalysts. World J. Microb. Biotechnol, 21:661-665.

Behera, B. (2009). Structural changes of FCC catalyst from fresh to regeneration stages and associated coke in a FCC refining unit: a multinuclearsolid state NMR approach. Catal. Today, 1 41:195-204.

Bhattacharyya, R. (2010). Know More about FCC. Catalysts. Bombay: Seminar Report 
Submitted as part of coursework for CL 618 (Catalysis and Surface Chemistry. Department of Chemical EngineeringIndian Institute of Technology.

Brunet, S. M. (2004). Review on the hydrodesulfurization of FCC gasoline: a review. Elsevier, 146-152.

Castells, X. (2012). Reciclaje de Residuos Industriales. Madrid: Ediciones Diaz de Santos. Segunda edición

Cerqueira, H., Caeiro, G., Costa, L., \& Ribeiro, F. (2008). Deactivation of FCC catalysts. Elsevier, 1-13.

Chen, H., Tseng, Y., \& Hsu, K. (2004). Spent FCC catalyst as a pozzolanic materialfor highperformance mortars. Cement Concrete Comp, 26:657-664.

Cherem da Cunha, A. (2011). A study of the particle size effect on the pozzolanic activity of anequilibrium catalyst. J. Therm. Anal. Calorim, 106:805-809.

Cho, S., Jung, K., \& Woo, S. (s.f.). Regeneration of spent RFCC catalyst irreversiblydeactivated by Ni, Fe, and V contained in heavy oil. . Appl. Catal., B: Environ, 33:249-261.

Dubois, R. (2005). Introducción a la Refinación del Petróleo. Su historia, la tecnología, su desarrollo, los productos y sus mercados, combustibles alternos, su economía. Buenos Aires: Eudeba.

Dweck, J. (2008). Study of a Brazilian spent catalyst ascement aggregate by thermal and mechanical analysis. J. Therm. Anal Cal, 92:121-127.

Dwyef, J. (1993). Fluid catalytic cracking: chemistry. Catalysis Today.Elsevier Science Publishers, 18:487-507.

Elvira, J. (1982). Los residuos sólidos y su aplicación en la construcción de carreteras. Carreteras, 3:8-17.

Enríquez, R. S. (2004). Simulación del regenerador de una unidad de desintegración catalítica fluidizada (FCC). Rev. Centro Inv. (Méx), Vol. 6, Núm. 21.

EPA. (1992). METHOD 1311. Toxicity characteristic leaching procedure. USA: Environmental Protection Agency.

Ferella, F. (2016). Review Oil refining spent catalysts: A review of possible recycling Technologies. Resources Conservation and Recycling, 108:10-20.

Fleitas, C. (2006). Informe Operacional. Refinería Esmeraldas. Fábrica Carioca de Catalizadores.FCC S.A. 
Floger, S. (2008). Elementos de la ingeniería de las reacciones químicas. México: Person Educación, Cuarta Edición.

García de Lomas, M. (2007). Pozzolanic reaction of aspent fluid catalytic cracking catalyst in FCC-cement mortars. J. Therm. Anal.Calorim, 90:443-447.

Gary, J., \& Handwerk, G. (2003). Refino de Petróleo: Tecnología y economía. Barcelona: Reverté.

Gonzalez, M. (2015). Síntesis y potenciales aplicaciones tecnológicas de materiales zeolíticos obtenidos a partir de catalizadores FCC agotados. Argentina: Tesis de Doctorado. Universidad Nacional de La Plata.

GRUNTEC. (2014). Instructivo para la toma de muestras. Environmental Services. Quito. Environmental Services. Quito.

Guisnet, M., \& Ribeiro, F. (2011). Deactivation and Regeneration of Zeolite Catalysts. Londres: Imperial College Press

Hudec, P. (2011). FCC Catalyst - Key element in refinery technology. Bratislava, Slovak Republic: 45th International Petroleum Conference, June 13, 2011.

INEN. (2000). Norma Técnica Ecuatoriana. NTE INEN 2288:2000. Productos Químicos Industriales Peligrosos. Etiquetado de Precaución. Quito-Ecuador: Instituto Ecuatoriano de Normalización. Registro Oficial No. 117 de Julio 11 del 2000.

INEN. (2013). Norma Técnica Ecuatoriana NTN INEN 2266:2013.Transporte, almacenamiento y manejo de materiales peligrosos. Ecuador: Instituto Ecuatoriano de Normalización.

Kent, J. (2012). Handbook of Industrial Chemistry and Biotechnology. New York: Springer.

Khang, S., \& Mosby, J. (2002). Catalyst deactivation due to deposition of reaction products in macropores during hydroprocessing of petroleum residuals. Ind. Eng. Chem. Process Des, 25:437-442.

Kugler, E., Dadyburjor, D., Moore, H., Stiller, A., \& Penn, J. (2001). Effect of Pretreat on the Performance of Metal Contaminated Commercial FCC Catalyst. Elsevier, 26-41.

Laespada, J. (1991). Tratamiento de residuos tóxicos y peligrosos (RTPs). Pasado, presente y Futuro. Ing. Quím, 23: 231-233.

Letzsch, W. (2014). Global demand for catalytic technology increases. Fuel HartEnergy Pub. Recuperado el 08 de Marzo de 2015, de http://www.hartfuel.com/f.catalyst.html

Levespiel, O. (. (2010). Ingeniería de las Reacciones Químicas. Oregon: Limusa Wiley, 3ra edición. 
Lloyd, L. (2011). Handbook of Industrial Catalysts. Fundamental and Aplied Catalysis. London: Springer.

MAE. (2004). Ley de Gestión Ambiental. . Ecuador: Ministerio del Ambiente. Codificación 19. Registro Oficial Suplemento 418 de 10 de septiembre de 2004.

MAE: Acuerdo No 026. (2008). Procedimiento para el Registro de Generadores de residuos Peligrosos. Ecuador: Ministerio del Ambiente. Publicado en el Registro Oficial No. 334 del 12 de mayo de 2008 .

MAE: Acuerdo Nº61. (2015). Reforma del libro VI del Texto Unificado de Legislación Secundaria. Ecuador: Ministerio del Ambiente. Año II - No 316 Quito, lunes 4 de mayo de 2015.

MAE: Acuerdo No 142. (2012). Listados nacionales de sustancias químicas peligrosas, desechos peligrosos y especiales. Ecuador: Ministerio del Ambiente. Publicado en el Registro Oficial No No 856 del 21 de diciembre de 2012.

MINAM. (2013). Guía para muestreo. Estándares de Calidad Ambiental (ECA). Perú: Dirección de Gestión Ambiental. Decreto Supremo No 002-2013.

Mitchell, M., Hoffman, J., \& Moore, H. (2003). Residual feed cracking catalyst. En J. Magee, \& M. Mitchell, Fluid Catalytic Cracking: Science and Technology (pags. 293-338). Amsterdam: Elsevier.

Morales, A. (2010). Characterization of wasted catalyst metallic products used in the oil industry. Avances en ciencias e ingeniería, 2:15-24.

NMX-AA-15-1985. (1992). Protección al Ambiente - Contaminación del suelo. Muestreo. Método de cuarteo. México: 6 de Noviembre de 1992.

NOM-052-SEMARNAT. (2005). Características, procedimiento de identificación, clasificación y listados de los residuos peligrosos. México: Norma publicada en el Diario Oficial de la Federación el 23 de junio de 2006

Olivares, J. (2007). Tratamiento y disposición final de residuos en PEMEX-refinación. México: Instituto Nacional de Ecología.

Pacewska, B. (2000). Hydration of cement slurry in the presence of spent cracking catalyst. Thermal Analysis and Calorimetry, 60:71-78.

Passamonti, J. D. (2008). Laboratory Evaluation of FCC Commercial Catalysts. Analysis of products of industrial importance. Catalysis Today, 314-318.

Perry, R. G. (2001). Manual del Ingeniero Químico de Perry. Zaragoza: Mc-Graw-Hill. 
Ramos, J. (2002). Estudio de la contaminación por metales pesados y otros procesos de degradación química en los suelos de invernadero del poniente almeriense. España: Tesis doctoral. Universidad de Almería.

RAOHE. (2001). Reglamento Sustitutivo al Reglamento Ambiental para Operaciones Hidrocarburíferas en el Ecuador, Decreto Ejecutivo 1215. Ecuador: Registro Oficial 265de 13 de Febrero del 2001.

REE. (2005). Catalíticas I, Manual de Operación Unidad Cracking Catalítico y Fraccionamiento. Esmeraldas: Refinería Estatal de Esmeraldas. Técnicas Reunidas S.A.

Sadeghbeigi, R. (2000). Fluid Catalytic Cracking Handbook: Design, Operation and Troubleshooting of FCC Facilities. Texas: Butterworth - Heinemann.

Salbilla, D., Kolmetz, K., Sim, C., \& Soyza, C. (2002). Fluidized Catalytic CrackerCatalyst Selection: Equilibrium Catalyst Quality and Considerations for Selections. USA.

Sánchez-Vilches, E. (1994). Aprovechamiento del catalizador usado de FCC de refinerías. España: Universtity Jaume I de Castelló.Tesis de Doctorado.

Scherzer, J. (1990). Octane-enhancing zeolitic FCC catalysts: scientific and technical aspects. New York: (1era. ed.)Marcel Dekker.

Sedrán, U. (2006). Curso FCC. Unidad de Capacitación de Petroecuador. Esmeraldas: KeyContac. Bienes y Servicios Técnicos.

Speight, J. (2007). The Chemistry and Technology of Petroleum. Taylor and Francis Group.

Strubinger, A., Morales, F., \& Aponte, K. (2014). Riesgo ambiental y uso de catalizador gastado de FCC en mezclas de morteros. Revista de la Facultad de Ingeniería U.C.V, 29: 93-106.

Tchobanoglous, G. (1977). Solid wastes: engineering principles and management issues. New York: McGraw-Hill.

Torres, N. (2010). Using spent fluid catalytic cracking (FCC) catalyst as pozzolanic addition. Ingeniería e investigación, 30:35-42.

TULSMA. (2003). Texto Unificado de Legislación Ambiental. Libro VI. Calidad Ambiental. Ecuador: Ministerio del Ambiente. Decreto Ejecutivo No 3516 del 31 de Marzo del 2003.

UOP. (2013). General Operation Manual.FCC Unit with VSS Reactor Combustor Regenerator Catalyst Cooler. Esmeraldas-Ecuador: Petroecuador.

USEPA. (1986). Test Methods for Evaluating Solid Waste, Physical/Chemical Methods. US Environmental Protection Agency. Recuperado el 25 de Enero de 2015, de 
www.epa.gov/epawaste/hazard/testmethods/sw846/online/index.htm

Vendrell, R. (1990). Gestión de los residuos industriales de Cataluña. Ing. Quím, 22:113-119.

Villaquirán, M., \& Mejía, R. (2015). Aprovechamiento del catalizador gastado de FCC en la síntesis de zeolitas de bajo contenido de sílice. Rev. Latin Am. Metal. Mat, 2:334-343.

Viswanathan, B. S. (2002). Catalysis: Principles and Applications. New Delhi: Narosa Publishing House.

Woltermann, G. M. (2003). Commercial preparation and characterization of FCC catalysts. En J. Magee, \& M. Mitchell, Fluid Catalytic Cracking: Science and Technology (págs. 105-144). Amsterdam: Elsevier.

Yoo, J. S. (1998). Metal recovery and rejuvenation of metal loaded spent catalyst. Elsevier Science, 28-34. 